\title{
CARACTERIZACIÓN CLÍNICO EPIDEMIOLÓGICA DEL SOBREPESO Y LA OBESIDAD EN LA PARROQUIA RURAL SAN GERARDO, CHIMBORAZO, ECUADOR
}

\author{
CLINICAL EPIDEMIOLOGICAL CHARACTERIZATION OF OVERWEIGHT AND \\ OBESITY IN THE SAN GERARDO RURAL PARISH, CHIMBORAZO, ECUADOR \\ Elda Valdés Gonzaléz ${ }^{(1)}$; Karolina Abad Urgiles ${ }^{(2)}$; \\ Fernanda Soria Granizo ${ }^{(2)}$; Urbano Solis Cartas ${ }^{(1,3)}$; Jorge Valdés González ${ }^{(3)}$.
}

(1) Universidad Nacional de Chimborazo, Riobamba - Ecuador.

(2) Ministerio Salud Pública del Ecuador, Zona 3, Riobamba - Ecuador.

(3) Escuela Superior Politécnica de Chimborazo, Riobamba - Ecuador.

Email:umsmwork74@gmail.com

https://doi.org/10.33789/talentos.8.2.156

\begin{abstract}
Resumen. Los trastornos nutricionales por exceso constituyen factores de riesgo para múltiples enfermedades crónicas. Su conocimiento puede ser utilizado como predictor de aparición o de complicaciones de las mismas. El objetivo de la presente investigación fue caracterizar desde el punto de vista clínico epidemiológico el sobrepeso y la obesidad en los adultos de la parroquia rural San Gerardo de la provincia Chimborazo. Para esto se desarrolló una investigación básica, no experimental, con diseño descriptivo que tuvo como universo un total de 414 personas y la muestra quedó conformada por 213 adultos con sobrepeso u obesidad. Se aplicó un cuestionario para obtener la información necesaria en relación a las variables identificadas para el estudio. Los principales resultados incluyen promedio de edad de 44,23 años y predominio de féminas (60,56\%). El 41,78\% de las personas reportó al menos una enfermedad, donde la hipertensión arterial (50,56\%), el hipotiroidismo (23,60\%) y la diabetes mellitus $(17,98 \%)$ fueron las más representadas; el 69,65\% de las personas presentó sobrepeso y el 30,05\% obesidad. El 70,89\% de las personas presentó un nivel bajo de conocimiento sobre prevención del sobrepeso y la obesidad. Se concluye que existe un elevado porcentaje de población adulta en la parroquia rural San Gerardo con trastornos nutricionales por exceso; lo que incrementa el riesgo de padecer enfermedades crónicas. Los resultados obtenidos en torno al nivel de conocimiento
\end{abstract}


sobre prevención del sobrepeso y la obesidad muestran la necesidad de implementar acciones educativas encaminadas a mejorar el conocimiento de la población sobre temas fundamentales de salud.

Palabras clave: Epidemiología, estado nutricional, obesidad, manifestaciones clínicas, sobrepeso.

Abstract. Excess nutritional disorders are risk factors for multiple chronic diseases. Their knowledge can be used as a predictor of their appearance or complications. The objective of this research was to characterize overweight and obesity in adults from the rural San Gerardo parish of Chimborazo province from the clinical epidemiological point of view. For this, a basic, non-experimental research was developed, with a descriptive design that had a total of 414 people as a universe and the sample was made up of 213 adults with overweight or obesity. A questionnaire was applied to obtain the necessary information in relation to the variables identified for the study. The main results include an average age of 44.23 years and a predominance of females (60.56\%). 41.78\% of the people reported at least one disease, where arterial hypertension (50.56\%), hypothyroidism (23.60\%) and diabetes mellitus (17.98\%) were the most represented; $69.65 \%$ of the people were overweight and $30.05 \%$ obese. $70.89 \%$ of the people presented a low level of knowledge about the prevention of overweight and obesity. It is concluded that there is a high percentage of the adult population in the rural San Gerardo parish with excessive nutritional disorders; which conditions a high morbidity due to chronic diseases. The results obtained regarding the level of knowledge on the prevention of overweight and obesity show the need to implement educational actions aimed at improving the population's knowledge on fundamental health issues.

Keyword: Epidemiology, nutritional condition, obesity, clinical manifestations, overweight.

\section{INTRODUCCIÓN}

Los trastornos nutricionales (TN) son señalados en la actualidad como una de los principales factores de riesgo para la aparición de distintas enfermedades crónicas no transmisibles (ECNT). Dentro de ellas destacan la hipertensión arterial (HTA), la diabetes mellitus (DM) y otras (Sapunar, et al, 2018). Desde el punto de vista clasificatorio los TN se subdividen en TN por defecto y por exceso; dentro de estos últimos se incluyen el sobrepeso y la obesidad (López Plaza, \& Bermejo López).
La obesidad y el sobrepeso se definen como una acumulación anormal o excesiva de grasa que puede ser perjudicial para la salud. Se reporta que en países desarrollados constituyen la primera causa de aparición de enfermedades cardio y cerebrovasculares, teniendo una elevada representatividad en la morbimortalidad por estas afecciones (Villena Chávez, 2017; Vicente Sánchez, et al, 2017).

En Ecuador los TN por exceso constituyen 
un serio problema de salud, según datos ofrecidos por el Instituto Nacional de Estadística y Censo (INEC) cerca del $47 \%$ de la población adulta sufre de sobrepeso u obesidad; un problema que también se detecta en población infantil, donde se llega a reportar más del $20 \%$ de niños menores de 12 años que sufren de obesidad infantil (Ortiz, et al, 2017; INEC, 2019).

En la parroquia rural de San Gerardo, perteneciente al cantón Guano, provincia Chimborazo, existe una elevada incidencia de hipertensión arterial y diabetes mellitus como forma de expresión de ECNT y que son consideradas en Ecuador como una de las principales causas de discapacidad, morbilidad y muerte (De la Rosa, \& Acosta Silva, 2017). Según datos reportados en el Análisis de la Situación de Salud del año 2019 la incidencia de HTA alcanza hasta el $31,16 \%$ de la población comprendida entre 18 y 60 años de edad; en el caso de la DM la incidencia se eleva hasta el $16,21 \%$ de la población en ese rango de edades. Esta misma fuente señala un $21,32 \%$ de la población adulta con obesidad y un $38,76 \%$ con sobrepeso (ASIS, 2019).

Existe relación directa entre HTA, DM, sobrepeso y la obesidad con factores comunes entre ellos (Wan, 2017). Destacan la inactividad física, los malos hábitos nutricionales y la conducta alimentaria inadecuada; por lo que incidir en estos factores constituye una herramienta viable para minimizar el riesgo de aparición de sobrepeso y obesidad y de esta forma de ECNT como la HTA y la DM.

Es por eso que teniendo en cuenta la elevada incidencia de HTA y DM en la población adulta de la parroquia rural San Gerardo, la repercusión que tienen estas enfermedades en la morbimortalidad general y el papel que juegan el sobrepeso y la obesidad como factores de riesgo de estas afecciones; se decide realizar esta investigación con el objetivo de caracterizar, clínica y epidemiológicamente, el sobrepeso y la obesidad presente en la población adulta de la parroquia rural San Gerardo del cantón Guano en la provincia Chimborazo.

\section{MATERIALES Y MÉTODOS}

Se realizó una investigación básica, no experimental, transversal, que incluyó un enfoque mixto al integrar elementos cualitativos y cuantitativos, cuyo alcance fue descriptivo y correlacional. El universo estuvo constituido por 474 personas adultas residentes en la parroquia rural San Gerardo durante el periodo comprendido entre julio y diciembre del año 2019.

Para realizar el cálculo del tamaño de la muestra se utilizó la fórmula matemática para poblaciones conocidas. Después de realizar el cálculo se determinó que la muestra debería estar constituida por un total de 213 personas; para conformar la muestra se utilizó el método de muestreo aleatorio simple donde todas las personas tuvieron las mismas posibilidades de formar parte del estudio. Cada una de las personas incluidas en la investigación cumplió los siguientes criterios de inclusión y exclusión definidos para la investigación. 


\section{Criterios de inclusión}

- Personas comprendidas entre $20 \mathrm{y}$ 60 años de edad residentes de forma permanente en la parroquia rural San Gerardo con diagnóstico de sobrepeso $\mathrm{u}$ obesidad confirmada en la historia clínica.

- Personas que expresaron, mediante la firma del consentimiento informado, su deseo de participar en el estudio.

Para el desarrollo del estudio se identificaron 3 variables de investigación. La primera de ellas se denominó características generales de las personas que incluyó las siguientes subvariables de investigación: edad, sexo, nivel educacional, autoidentificación étnica, presencia de enfermedades y tipo de enfermedades. Otra variable fue la denominada como estado nutricional (EN) y la tercera y última variable fue denominada como nivel de conocimiento relacionado con sobrepeso y la obesidad.

Como técnicas de investigación se utilizó la revisión documental y la entrevista. La revisión documental permitió obtener información actualizada sobre el sobrepeso, la obesidad y sus implicaciones sobre las ECNT. La entrevista acompañó la aplicación del cuestionario de investigación; permitió obtener información relacionada con los objetivos del estudio y aclarar dudas en los participantes relacionados con los objetivos y métodos utilizados en la investigación.

El instrumento de investigación utilizado fue un cuestionario diseñado específicamente para el estudio que estuvo conformado por un total de 17 preguntas divididas en 2 sesiones. La primera sesión incluyó un total de 7 preguntas orientadas a la identificación de las características clínico epidemiológicas de los adultos incluidos en el estudio. La segunda sesión estuvo constituida por 10 preguntas orientadas a identificar el nivel de conocimiento de las personas participantes en el estudio sobre prevención y complicaciones del sobrepeso y la obesidad.

El cuestionario de investigación fue sometido a la revisión de 7 expertos, especialistas en medicina interna, promoción de salud, educación, metodología de investigación y medicina general. Después de una segunda ronda de revisión los expertos opinaron favorablemente en torno a su aplicación. Antes de la aplicación definitiva del instrumento se aplicó una prueba piloto en 13 personas que permitió identificar errores semánticos que pudieran estar presentes.

La determinación del nivel de conocimiento relacionado con el sobrepeso y la obesidad en los participantes se basó en el resultado del cuestionario de investigación; para lo que se adoptó la siguiente clasificación:

- Menos de 5 respuestas positivas: nivel bajo de conocimiento

- Entre 5 y 7 respuestas positivas: nivel medio de conocimiento

- Más de 7 respuestas positivas: nivel elevado de conocimiento

Para determinar el EN de los participantes se utilizó el resultado del índice de masa corporal (IMC). Para determinar el IMC se procedió a dividir el peso de las personas, expresado en kilogramos, sobre la talla en centímetros cuadrados. Los parámetros de 
referencia utilizados fueron los definidos por la Organización Mundial de la Salud (OMS, s.f.):

- Bajo peso: IMC inferior a 18,5 puntos

- Normo peso: IMC entre 18,5 y 24,9 puntos

- Sobre peso: IMC entre 25,0 y 29,9 puntos

- Obesidad: IMC igual o superior a 30 puntos

Con los datos obtenidos se procedió a realizar el procesamiento estadístico de forma automatizada con la ayuda del programa SPSS en su versión 26,0 para Windows. Se determinaron medidas de tendencia central y de dispersión para procesar las variables cuantitativas y frecuencias absolutas $y$ porcentajes en el procesamiento de las variables cualitativas. Se definió el nivel de confianza en el $95 \%$, el margen de error en el $5 \%$ y la significación estadística en una $p$ $\leq 0,05$. Los resultados fueron expresados en forma de tablas estadísticas para facilitar la comprensión de los mismos.

Durante el desarrollo de la investigación se cumplieron con las normas y procedimientos establecidos en la Declaración de Helsinki II para realizar investigaciones en seres humanos. Todos los participantes fueron informados previamente de los objetivos y métodos del estudio; la incorporación de los mismos se realizó de manera voluntaria y posterior a la firma del consentimiento informado. La investigación no generó costos económicos para los participantes. Los datos obtenidos fueron utilizados únicamente con fines investigativos y la base de datos con toda la información recopilada fue eliminada después de realizado el informe final del estudio.

Adicionalmente se cumplieron las medidas orientadas por el COE nacional y provincial mediante acciones puntuales encaminadas a prevenir el contagio por la COVID-19. Las acciones implementadas incluyeron el uso obligatorio de mascarillas, lavado frecuente de manos, uso de alcohol y gel antibacterial y mantener el debido distanciamiento.

\section{RESULTADOS Y DISCUSIÓN}

La tabla 1 muestra la distribución de personas según las características generales. Destaca un promedio de edad de 44,23 años con predominio de personas entre 41 y 50 años $(41,78 \%)$. En relación al sexo predominó el sexo femenino con el $60,56 \%$, en relación a las personas del sexo masculino $(39,44 \%)$. El $57,75 \%$ de los participantes en el estudio refirió como nivel educacional secundaria terminada, el 31,46\% refirió haber vencido los estudios primarios y el 5,16\% refirió haber culminado estudios de educación superior. El $73,24 \%$ se auto identificó como mestizos(as), el $13,62 \%$ se auto identificó como indígenas y el $9,86 \%$ como blancos(as). Solo el 3,28\% de las personas se auto identificó como negros(as).

El análisis de la presencia de enfermedades en estas personas mostró que el $41,78 \%$ de ellos expresó tener diagnóstico de al menos una ECNT. Dentro de estas el mayor porciento de referencia fue para la HTA $(50,56 \%)$, seguido del hipotiroidismo $(23,60 \%)$ y de la DM (17,98\%). Otras afecciones que también fueron referidas fueron la artritis reumatoide 
$(6,74 \%)$, la gota y la insuficiencia cardiaca con idéntico 3,37\%. La epilepsia y la cardiopatía isquémica fueron referidas por dos personas en cada caso (2,25\%). El $69,85 \%$ de las personas investigadas presentaron sobrepeso y el 30,05 obesidad.

Tabla I. Distribución de personas según características generales.

\begin{tabular}{lc}
\hline \multicolumn{1}{c}{$\begin{array}{c}\text { Características } \\
\text { generales }\end{array}$} & $\begin{array}{c}\text { Muestra total de } \\
\mathbf{2 1 3} \text { personas } \\
\text { Frecuencia (por } \\
\text { ciento) }\end{array}$ \\
\hline $\begin{array}{l}\text { Promedio de edad } \\
\text { (años) }\end{array}$ & $44,23{ }^{*} \mathrm{DE} 18,77$ \\
\end{tabular}

\section{Edad}

Entre 20 y 30 años

$18(8,45)$

Entre 31 y 40 años

$57(26,76)$

Entre 41 y 50 años

$89(41,78)$

Entre 51 y 60 años

$49(23,01)$

Sexo

Masculino

$84(39,44)$

Femenino

$129(60,56)$

\section{Nivel educacional}

$\begin{array}{ll}\text { Analfabeto } & 12(5,63) \\ \text { Primaria } & 67(31,46) \\ \text { Secundaria } & 123(57,75) \\ \text { Superior } & 11(5,16)\end{array}$

Autoidentificación étnica

$\begin{array}{ll}\text { Blanco(a) } & 21(9,86) \\ \text { Mestizo(a) } & 156(73,24) \\ \text { Negro(a) } & 7(3,28) \\ \text { Indígena } & 29(13,62)\end{array}$

Presencia de enfermedades

$\begin{array}{ll}\mathrm{Si} & 89(41,78) \\ \mathrm{No} & 124(58,22)\end{array}$

Tipo de enfermedades $n=89$ personas

$\begin{array}{ll}\text { Hipertensión arterial } & 45(50,56) \\ \text { Diabetes mellitus } & 16(17,98) \\ \text { Hipotiroidismo } & 21(23,60) \\ \text { Artritis reumatoide } & 6(6,74) \\ \text { Gota } & 3(3,37) \\ \text { Epilepsia } & 2(2,25)\end{array}$

$\begin{array}{ll}\text { Cardiopatía isquémica } & 2(2,25) \\ \text { Insuficiencia cardiaca } & 3(3,37)\end{array}$

Estado Nutricional

\begin{tabular}{ll} 
Sobrepeso & $149(69,65)$ \\
Obesidad & $64(30,05)$ \\
\hline
\end{tabular}

Fuente: cuestionario de investigación

La tabla 2 muestra el comportamiento del nivel de conocimiento relacionado con el sobrepeso y la obesidad de las personas que participaron en la investigación. Se observa un predominio de personas con nivel bajo de conocimiento $(70,89 \%)$, dato que fue estadísticamente significativo. Le siguieron las personas con nivel medio $(23,00 \%)$ y solo el $6,13 \%$ de las personas alcanzó un nivel de conocimiento elevado.

Tabla II. Distribución de personas según nivel de conocimientos relacionado con el sobrepeso y la obesidad en el pretest.

\begin{tabular}{l|l|l|l}
\hline $\begin{array}{l}\text { Nivel de } \\
\text { conocimiento } \\
\text { sobrepeso y } \\
\text { obesidad }\end{array}$ & Frecuencia & $\begin{array}{l}\text { Por } \\
\text { ciento }\end{array}$ & ${ }^{*} \mathbf{p}$ \\
\hline Bajo & 151 & 70,89 & 0,029 \\
Medio & 49 & 23 & 0,077 \\
Alto & 13 & 6,13 & 0,094 \\
\hline
\end{tabular}

Fuente: cuestionario de investigación $\quad * p \leq 0,05$

\section{Discusión}

El análisis de los resultados obtenidos en la investigación aporta algunos elementos destacados. El primero de ellos es el elevado por ciento de personas con al menos una ECNT identificada. A pesar de que no existen reportes previos para comparar este resultado, el equipo de investigación considera importante aportar algunas consideraciones al respecto. 
Varios elementos pueden justificar el elevado por ciento de ECNT en la población de la parroquia rural San Gerardo. El primero de ellos es relacionado con la poca noción de riesgo de salud imperante en la población. Esta situación está motivada por el inadecuado funcionamiento de los servicios de salud en la parroquia; donde la actividad salubrista se centra principalmente en la medicina curativa, obviando la medicina epidemiológica basada en la promoción de salud y la prevención de enfermedades (Monroy Antón, et al, 2018; Olivos Chuquino, \& Rojas Jaimes, 2020).

El patrón de presencia de ECNT con predominio de HTA se centra en el poco control de los factores de riesgos de esta enfermedad motivados por los elementos antes mencionados. Existen condiciones como son las costumbres alimentarias inadecuadas y la poca frecuencia de realización de actividades físicas (Chacón O'Farrill, \& Cortes, 2018). Según los datos contenidos en el Análisis Situacional de Salud se describe que en la comunidad rural San Gerardo es casi nula la práctica de actividades físicas, solamente el $16,3 \%$ de las personas residentes realizan algún tipo de actividad física; y esta se centra solamente en partidos de futbol entre personas jóvenes (ASIS, 2019).

La presencia de hipotiroidismo enun por ciento superior a la media de otras comunidades se debe a una característica predominante en el contexto de la investigación; una posible explicación no comprobada científicamente está dada por la ausencia de iodo en la sal de consumo; entendiendo que el iodo juega un papel fundamental en el adecuado funcionamiento de la glándula tiroides (Garcés Salazar, Paredes Cuesta, \& Martínez
Gualpa, 2019).

Uno de los elementos fundamentales del estudio fue determinar el nivel de conocimiento de las personas adultas relacionado con el sobrepeso y la obesidad. El bajo nivel de conocimiento predominante es un reflejo de lo expuesto anteriormente en relación al inadecuado funcionamiento de la medicina preventiva en la parroquia. Autores como Zamora Niño y colaboradores (2019) consideran que elevar el nivel de conocimiento sobre la salud personal y colectiva constituye el elemento fundamental para prevenir la propagación de enfermedades transmisibles y el control de factores de riesgo de ECNT (Rico Sánchez, 2018).

Sin embargo, la responsabilidad de realizar actividades encaminadas a elevar el nivel de conocimiento de los pobladores no es solamente de los profesionales de la salud. Todas las organizaciones públicas, privadas y sociales tienen su cuota de responsabilidad y deben de coordinar esfuerzos en pos de la consolidación de este tipo de actividades.

Las intervenciones educativas, según Burgo Bencomo y colaboradores (2019); han sido señaladas como estrategias efectivas que permiten de una forma rápida, sencilla $y$ económica elevar el nivel de conocimiento de determinado grupo poblacional sobre temas de salud generales y específicos.

Algunos ejemplos son los reportes realizados por Sanchez y colaboradores (2017) quienes señalan al aumento del nivel de conocimiento sobre alimentación en las personas investigadas. También Macia Ferreiro y colaboradores (2020) reportan resultados favorables en el conocimiento de prevención de alcoholismo en adolescentes. 
Los resultados de esta investigación y los reportes de la literatura muestran la necesidad de implementar acciones educativas encaminadas a lograr el aumento del nivel de conocimiento de la población sobre temas de salud importantes como es el sobrepeso y la obesidad.

\section{CONCLUSIONES}

Se identificó un elevado porcentaje de personas adultas, residentes en la parroquia rural San Gerardo, con trastornos nutricionales por exceso; situación que condiciona una elevada morbilidad de ECNT. El nivel de conocimiento predominante sobre prevención del sobrepeso y la obesidad es bajo; por lo que se considera necesario implementar acciones educativas encaminadas a dar un vuelco al desconocimiento que sobre temas fundamentales de salud existe en la población adulta.

\section{REFERENCIAS BIBLIOGRÁFICAS}

Análisis Situacional de Salud (ASIS). (2019). Análisis de la situación de salid parroquia San Gerardo. Guano, Chimborazo, Ecuador.

Burgo Bencomo, O.B., León González, J.L., Cáceres Mesa, M.L., Pérez Maya, C.J., \& Espinoza Freire, E.E. (2019). Some thoughts on research and educational intervention. Revista Cubana de Medicina Militar, 48(Supl. 1), e383. Recuperado de http://scielo.sld.cu/scielo. php? script $=$ sci_arttext\&pid $=$ S0138$\underline{65572019000500003 \& \operatorname{lng}=\text { es\&tlng= }}$ $\underline{\text { en }}$

Chacón O’Farrill, D., \& Cortes A., (2018). Intervención educativa del síndrome de flujo vaginal en gestantes. Revista Cubana de Obstetricia y Ginecología, 44(2), 1-13. Recuperado en 08 de mayo de 2021, de http://scielo.sld.cu/scielo. php? script $=$ sci_arttext\&pid $=$ S0138-

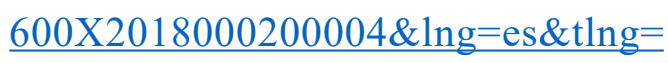
$\underline{\text { es }}$

De la Rosa, J.M., \& Acosta Silva, M. (2017). Posibles factores de riesgo cardiovasculares en pacientes con hipertensión arterial en tres barrios de Esmeraldas, Ecuador. Revista Archivo Médico de Camagüey, 21(3), 361-369. Recuperado de http://scielo.sld.cu/scielo. php? script $=$ sci arttext\&pid $=\mathrm{S} 1025$ $\underline{02552017000300007 \& \operatorname{lng}=\mathrm{es} \& \operatorname{tlng}=\mathrm{es}}$

Garcés Salazar, M.C., Paredes Cuesta, D.M., \& Martínez Gualpa, L.R.. (2019). Relación entre hipotiroidismo $\mathrm{y}$ artritis reumatoide. Revista Cubana de Reumatología, 21(2), e86. Recuperado de http://scielo.sld.cu/scielo. php? script $=$ sci_arttext\&pid $=\mathrm{S} 1817$ 59962019000200002\&lng=es\&tlng=es

Instituto Nacional de Estadística y Censo (INEC). 2019. Trastornos nutricionales en la población ecuatoriana. Quito, Ecuador.

López Plaza, B., \& Bermejo López, L.M. (2017). Nutrición y trastornos del sistema inmune. Nutrición Hospitalaria, 34(Supl. 4), 68-71. Recuperado de https://dx.doi.org/10.20960/nh.1575 
Macías Ferreiro, K., Vinces Ubillus, M.A., Mendoza Cedeño, I.G., Briones Bermeo, N.P., \& Mera Leones, F.B. (2020). Educational intervention for the prevention of alcoholism in adolescents in Ecuador schools. Revista de Ciencias Médicas de Pinar del Río, 24(1), 86-95. Recuperado de $\quad$ http://scielo.sld.cu/scielo. php? script $=$ sci_arttext\&pid $=\mathrm{S} 1561$ $\underline{31942020000100086 \& \operatorname{lng}=\text { es\&tlng= }}$ $\underline{\text { en }}$

Monroy Antón, A., Calero Morales, S., \& Fernández Concepción, R.R. (2018). Los programas de actividad física para combatir la obesidad y el sobrepeso en adolescentes. Revista Cubana de Pediatría, 90(3), 1-9. Recuperado de http://scielo.sld.cu/scielo. php? script $=$ sci_arttext\&pid $=$ S0034$\underline{75312018000300016 \& \operatorname{lng}=\mathrm{es} \& \ln \mathrm{ln}=\mathrm{es}}$

Olivos-Chuquino, S., \& Rojas-Jaimes, J.E. (2020). Eficacia de los programas educativos para reducir el sobrepeso y obesidad en niños. Revista Medica Herediana, 31(2), 132-133. Recuperado de https://dx.doi.org/10.20453/rmh. $\underline{\text { v3 } 1 \mathrm{i} 2.3778}$

Organización Mundial de la Salud (OMS). (s.f.). Datos y Cifras. 10 Datos sobres la Obesidad. Recuperado de https:// www.who.int/features/factfiles/ obesity/facts/es/\#: : text $=\mathrm{E} 1 \% 20$ $\%$ C3\%ADndice $\% 20 \mathrm{de} \% 20$ masa $\% 20$ corporal,igual $\% 200 \% 20$ superior $\% 20$ $\underline{\mathrm{a} \% 2030}$

Ortiz, R., Torres, M., Peña Cordero, S., Palacio
Rojas, M., Crespo, J.A., Sánchez, J.F., \& Pineda Álvarez, D., et al. (2017). Comportamiento epidemiológico de la obesidad y factores de riesgo asociados en la población rural de Cumbe, Ecuador. Archivos Venezolanos de Farmacología y Terapéutica, 36(3), 88-96. Recuperado de $\quad$ http://ve.scielo.org/scielo. php? $\mathrm{script}=$ sci_arttext\&pid $=\mathrm{S} 0798$ $\underline{02642017000300006 \& \operatorname{lng}=\mathrm{es} \& \mathrm{t} \operatorname{lng}=\mathrm{pt}}$

Rico Sánchez, R., Juárez Lira, A., Sánchez Perales, M., \& Muñoz Alonso, L.R. (2018). Nivel de Conocimientos, Estilos de Vida y Control Glicémico en Pacientes con Diabetes Mellitus tipo 2. Ene, 12(1), 757. Recuperado de http:// scielo.isciii.es/scielo.php?script $=\mathrm{sci}$ arttext\&pid $=$ S1988-348X2018000100 $\underline{006 \& \operatorname{lng}=\mathrm{es} \& \mathrm{t} \operatorname{lng}=\mathrm{es}}$

Sánchez, V., Aguilar, A., González, F., Esquius, L., \& Vaqué, C. (2017). Evolución en los conocimientos sobre alimentación: una intervención educativa en estudiantes universitarios. Revista chilena de nutrición, 44(1), 19-27. Recuperado de https://dx.doi.org/10.4067/S0717$\underline{75182017000100003}$

Sapunar, J., Aguilar-Farías, N., Navarro, J., Araneda, G., Chandia-Poblete, D., Manríquez, V., Brito, R., \& Cerda, A. (2018). Alta prevalencia de trastornos nutricionales por exceso, resistencia insulínica y síndrome metabólico en escolares de la comuna de Carahue, Región de la Araucanía. Revista médica de Chile, 146(9), 978-986. Recuperado de https://dx.doi.org/10.4067/s0034$\underline{98872018000900978}$ 
Vicente Sánchez, B., García, K., González Hermida, A., \& Saura Naranjo, C.E. (2017). Sobrepeso y obesidad en niños de 5 a 12 años. Revista Finlay, 7(1), 47-53. Recuperado en 07 de mayo de 2021, de http://scielo.sld.cu/scielo. php? script $=$ sci_arttext\&pid $=$ S2221$\underline{24342017000100007 \& \operatorname{lng}=\text { es\&tlng }=\text { es }}$

Villena Chávez, J.E. (2017). Prevalencia de sobrepeso y obesidad en el Perú. Revista Peruana de Ginecología y Obstetricia, 63(4), 593-598. Recuperado de http://www.scielo.org.pe/scielo. php?script $=$ sci_arttext\&pid $=$ S2304$\underline{51322017000400012 \& \operatorname{lng}=\text { es\&tlng }=\text { es }}$

Wan, L., Aozi, F., Lesly Solís, A., \& Fernández-Britto Rodríguez, J.E.. (2017). Influencia del tabaquismo, la hipertensión arterial y la diabetes mellitus en las enfermedades oftalmológicas. Revista Cubana de Oftalmología, 30(3), 1-14. Recuperado de $\quad$ http://scielo.sld.cu/scielo. php? script $=$ sci_arttext\&pid $=$ S0864$\underline{21762017000300010 \& \operatorname{lng}=\mathrm{es} \& \operatorname{tlng}=\mathrm{es}}$

Zamora-Niño, C.F., Guibert-Patiño, A.L., De La Cruz-Saldaña, T., TicseAguirre, R., \& Málaga, G. (2019). Evaluación de conocimientos sobre su enfermedad en pacientes con diabetes tipo 2 de un hospital de Lima, Perú y su asociación con la adherencia al tratamiento. Acta Médica Peruana, 36(2), 96-103. Recuperado de http://www.scielo.org.pe/scielo. php? script $=$ sci_arttext\&pid $=\mathrm{S} 1728$ $\underline{59172019000200004 \& \operatorname{lng}=\mathrm{es} \& \mathrm{t} \operatorname{lng}=\mathrm{es}}$ 